\title{
A Collectively Fixed Point Theorem in Abstract Convex Spaces and Its Applications
}

\author{
Haishu $\mathrm{Lu}^{1}$ and Qingwen $\mathrm{Hu}^{2}$ \\ ${ }^{1}$ School of Business, Jiangsu University of Technology, Changzhou, Jiangsu 213001, China \\ ${ }^{2}$ Department of Mathematical Sciences, University of Texas at Dallas, Richardson, TX 75080, USA \\ Correspondence should be addressed to Haishu Lu; luhaishu@126.com
}

Received 1 April 2013; Revised 11 June 2013; Accepted 16 June 2013

Academic Editor: Stanislav Hencl

Copyright (c) $2013 \mathrm{H}$. Lu and Q. Hu. This is an open access article distributed under the Creative Commons Attribution License, which permits unrestricted use, distribution, and reproduction in any medium, provided the original work is properly cited.

\begin{abstract}
The main purpose of this paper is to establish a new collectively fixed point theorem in noncompact abstract convex spaces. As applications of this theorem, we obtain some new existence theorems of equilibria for generalized abstract economies in noncompact abstract convex spaces.
\end{abstract}

\section{Introduction}

Collectively fixed point theorems for a family of set-valued mappings play a vital role in studying various nonlinear problems. In 1991, Tarafdar [1] established a collectively fixed point theorem in topological vector spaces and gave applications to mathematical economies, game theory, and problems of social sciences. Since then, a lot of generalizations and applications of collectively fixed point theorem under different assumptions and different underlying spaces have been studied by many authors (see [2-7] and the references therein).

Inspired and motivated by the above results, in this paper, we establish a new collectively fixed point theorem in noncompact abstract convex spaces. As applications of this fixed point theorem, some new existence theorems of equilibria for generalized abstract economies are proved under the setting of noncompact abstract convex spaces.

\section{Preliminaries}

Let $X$ be a set. We will denote by $2^{X}$ the family of all subsets of $X$, by $\langle X\rangle$ the family of nonempty finite subsets of $X$. Let $A$ be a subset of a topological space $X$; we will denote the interior and the closure of $A$ by int $A$ and $\operatorname{cl} A$, respectively. Let $X$ and $Y$ be two nonempty sets and $T: X \rightarrow 2^{Y}$ a set-valued mapping.
Then the set-valued mapping $T^{-1}: Y \rightarrow 2^{X}$ is defined by $T^{-1}(y)=\{x \in X: y \in T(x)\}$ for each $y \in Y$.

Definition 1 (see [8]). An abstract convex space $(E, D ; \Gamma)$ consists of a topological space $E$, a nonempty set $D$, and a set-valued mapping $\Gamma: D \rightarrow 2^{E}$ with nonempty values. One may denote $\Gamma_{A}:=\Gamma(A)$ for each $A \in\langle D\rangle$.

Let $(E, D ; \Gamma)$ be an abstract convex space. For any $D^{\prime} \subset D$, the $\Gamma$-convex hull of $D^{\prime}$ is denoted and defined by

$$
\operatorname{co}_{\Gamma}\left(D^{\prime}\right):=\bigcup\left\{\Gamma_{A}: A \in\left\langle D^{\prime}\right\rangle\right\} \subseteq E
$$

(co is reserved for the convex hull in vector spaces). A subset $X$ of $E$ is called a $\Gamma$-convex subset of $(E, D ; \Gamma)$ relative to $D^{\prime}$ if, for each $N \in\left\langle D^{\prime}\right\rangle$, we have $\Gamma_{N} \subseteq X$; that is, $\operatorname{co}_{\Gamma}\left(D^{\prime}\right) \subseteq$ $X$. This means that $\left(X, D^{\prime} ;\left.\Gamma\right|_{\left\langle D^{\prime}\right\rangle}\right)$ itself is an abstract convex space called a subspace of $(E, D ; \Gamma)$. When $D \subseteq E$, the space is denoted by $(E \supseteq D ; \Gamma)$. In such case, a subset $X$ of $E$ is said to be $\Gamma$-convex if $\operatorname{co}_{\Gamma}(X \bigcap D) \subseteq X$; in other words, $X$ is $\Gamma$-convex relative to $D^{\prime}:=D \bigcap X$. In case $E=D$, let $(E ; \Gamma):=(E, E ; \Gamma)$.

Remark 2. There are a lot of examples of abstract convex spaces; see [7-13] and references therein. Here, for convenience, we give the following three examples of abstract convex spaces which are cited in this paper.

(a) Let $X$ be a topological space and $\left\{F_{A}\right\}$ be a given family of nonempty contractible subsets of $X$ indexed by 
$A \in\langle X\rangle$ such that $F_{A} \subseteq F_{B}$ whenever $A \subseteq B$. The couple $\left(X, F_{A}\right)$ is called an $H$-space (see [14]). A set $D \subseteq X$ is said $H$-convex if $F_{A} \subseteq D$ for each $A \in\langle D\rangle$. A set $K \subseteq X$ is said $H$-compact if, for each $A \in\langle X\rangle$, there is a compact $H$-convex set $D \subseteq X$ such that $K \bigcup A \subseteq D$.

(b) A generalized convex space or a $G$-convex space $(X, D ; \Gamma)($ see $[15])$ consists of a topological space $X$ and a nonempty set $D$ such that, for each $A \in\langle D\rangle$ with the cardinality $|A|=n+1$, there exist a subset $\Gamma(A)$ of $X$ and a continuous function $\varphi_{A}: \Delta_{n} \rightarrow$ $\Gamma(A)$ such that $J \in\langle A\rangle$ implies $\varphi_{A}\left(\Delta_{J}\right) \subseteq \Gamma(J)$. Here, $\Delta_{n}$ is the standard $n$-simplex with vertices $\left\{e_{i}\right\}_{i=0}^{n}$ and $\Delta_{J}$ the face of $\Delta_{n}$ corresponding to $J \in\langle A\rangle$; that is, if $A=\left\{a_{0}, a_{1}, \ldots, a_{n}\right\}$ and $J=\left\{a_{i_{0}}, \ldots, a_{i_{k}}\right\} \subseteq A$, then $\Delta_{J}=\operatorname{co}\left\{e_{i_{0}}, e_{i_{1}}, \ldots, e_{i_{k}}\right\}$.

(c) A semilattice (see [16]) is a partially ordered set $X$, with the partial ordering denoted by $\leq$, for which, any pair $\left(x, x^{\prime}\right)$ of elements has a least upper bound, denoted by $x \vee x^{\prime}$. A topological semilattice is a topological space $X$ with a partial ordering $\leq$ for which it is a semilattice with a continuous sup operation; that is, the function $X \times X \rightarrow X,\left(x, x^{\prime}\right) \rightarrow x \vee x^{\prime}$ is continuous.

It is evident that each nonempty finite subset $A$ of a semilattice has a least upper bound, denoted by $\sup A$. In a partially ordered set $(X, \leq)$, two arbitrary elements $x$ and $x^{\prime}$ do not have to be comparable. In case $x \leq x^{\prime}$, the set $\left[x, x^{\prime}\right]=\left\{y \in X: x \leq y \leq x^{\prime}\right\}$ is called an order interval. Now assume that $(X, \leq)$ is a semilattice and $A$ is a nonempty finite subset of $X$. Then the set $\Delta(A):=\bigcup_{a \in A}[a$, sup $A]$ is well defined. A subset $D$ of $X$ is called $\Delta$-convex if, for any $N \in\langle D\rangle$, we have $\Delta(N) \subseteq D$.

Definition 3 (see [13]). Let $(E, D ; \Gamma)$ be an abstract convex space and $Z$ a set. For a set-valued mapping $F: E \rightarrow 2^{Z}$ with nonempty values, if a set-valued mapping $G: D \rightarrow 2^{Z}$ satisfies $F\left(\Gamma_{A}\right) \subseteq G(A)$ for each $A \in\langle D\rangle$, then $G$ is called a KKM map with respect to $F$. A KKM map $G: D \rightarrow 2^{E}$ is a KKM map with respect to the identity map $1_{E}$.

A set-valued mapping $F: E \rightarrow 2^{Z}$ is said to have the KKM property and called a $\mathfrak{\Omega}$-map, if, for any KKM map $G$ : $D \rightarrow 2^{Z}$ with respect to $F$, the family $\{G(y): y \in D\}$ has the finite intersection property. We denote

$$
\Re(E, D, Z):=\left\{F: E \longrightarrow 2^{Z} \mid F \text { is a } \Re \text {-map }\right\} .
$$

Let $Z$ be a topological space. A set-valued mapping $F$ : $E \rightarrow 2^{Z}$ is called a $\mathfrak{K} \mathbb{C}$-map (resp., $\mathfrak{\Re} \mathfrak{D}$-map) if, for any closed-valued (resp., open-valued) KKM map $G: D \rightarrow$ $2^{Z}$ with respect to $F$, the family $\{G(y): y \in D\}$ has the finite intersection property. In this case, we denote $F \in$ $\mathfrak{\Re} \mathfrak{C}(E, D, Z)($ resp., $F \in \mathfrak{N D}(E, D, Z))$. When $E=D$, we will write $\mathfrak{\Re} \mathfrak{C}(E, Z)$ (resp., $\mathfrak{\Re D}(E, Z)$ ) instead of $\mathfrak{\Re} \mathfrak{C}(E, D, Z)$ (resp., $\mathfrak{\Re D}(E, D, Z))$. Note that if $Z$ is a discrete space, then three classes $\mathfrak{\Re}, \mathfrak{I C}$, and $\mathfrak{K D}$ are identical. For more details, we refer to $[12,13,17,18]$ and the references therein.
Definition 4 (see $[17,18]$ ). The partial KKM principle for an abstract convex space $(E, D ; \Gamma)$ is the statement $1_{E} \in$ $\mathfrak{\Re} \mathfrak{S}(E, D, E)$; that is, for any closed-valued KKM map $G$ : $D \rightarrow 2^{E}$, the family $\{G(y): y \in D\}$ has the finite intersection property. The KKM principle is the statement that the same property also holds for any open-valued KKM map (i.e., $1_{E} \in$ $\mathfrak{\Re}(E, D, E) \bigcap \mathfrak{\Re} \mathfrak{D}(E, D, E))$.

An abstract convex space is called a (partial) KKM space if it satisfies the (partial) KKM principle. A lot of examples of (partial) KKM spaces can be found in $[9,17,18]$ and the references therein.

Definition 5 (see [19]). Let $X$ be a topological space, $Y$ a nonempty set, and $T: X \rightarrow 2^{Y}$ a set-valued mapping. $T$ is said to have local intersection property if, for each $x \in X$ with $T(x) \neq \emptyset$, there exists an open neighborhood $N(x)$ of $x$ such that $\bigcap_{z \in N(x)} T(z) \neq \emptyset$.

Remark 6. By Proposition 1 in Lin [19] and Lemma 3.1 in Llinares [20], we can see that the following conditions are equivalent.

(i) $T$ has the local intersection property and, for all $x \in$ $X, T(x)$ is nonempty;

(ii) $X=\bigcup_{y \in Y}$ int $T^{-1}(y)$;

(iii) for each $y \in Y, T^{-1}(y)$ contains an open subset $O_{y}$ of $X$ and $X=\bigcup_{y \in Y} O_{y}$.

Lemma 7 (see [8]). Let $\left\{\left(E_{i}, D_{i} ; \Gamma_{i}\right)\right\}_{i \in I}$ be a family of abstract convex spaces, where $I$ is an index set. Let $E:=\prod_{i \in I} E_{i}$ be equipped with the product topology and $D:=\prod_{i \in I} D_{i}$. For each $i \in I$, let $\pi_{i}: D \rightarrow D_{i}$ be the projection. Define $\Gamma=\prod_{i \in I} \Gamma_{i}:\langle D\rangle \rightarrow 2^{E}$ by $\Gamma(A):=\prod_{i \in I} \Gamma_{i}\left(\pi_{i}(A)\right)$ for each $A \in\langle D\rangle$. Then $(E, D ; \Gamma)$ is an abstract convex space.

Lemma 8 (see $[13])$. Let $(E, D ; \Gamma)$ be an abstract convex space, $\left(X, D^{\prime} ;\left.\Gamma\right|_{\left\langle D^{\prime}\right\rangle}\right)$ a subspace of $(E, D ; \Gamma)$, and $Z$ a topological space. If $F \in \mathfrak{\Re} \mathfrak{C}(E, D, Z)$, then $\left.F\right|_{X} \in \mathfrak{\Re} \mathfrak{C}\left(X, D^{\prime}, \operatorname{cl}(F(X))\right)$.

\section{A Collectively Fixed Point Theorem}

The following lemma is a special case of Theorem 11 in Park [12].

Lemma 9. Let $(X ; \Gamma)$ be an abstract convex space with $1_{X} \in$ $\mathfrak{\Re} \mathfrak{C}(X, X)$ (i.e., $(X ; \Gamma)$ satisfies the partial KKM principle). Let $S, T: X \rightarrow 2^{X}$ be two set-valued mappings satisfying the following conditions:

(i) for each $x \in X, \operatorname{co}_{\Gamma} S(x) \subseteq T(x)$ (i.e., for each $M \in$ $\langle S(x)\rangle, \Gamma(M) \subseteq T(x))$;

(ii) $S^{-1}$ has open values;

(iii) there exists $\left\{\bar{y}_{0}, \ldots, \bar{y}_{n}\right\} \in\langle X\rangle$ such that $X=$ $\bigcup_{j=0}^{n} S^{-1}\left(\bar{y}_{j}\right)$.

Then $T$ has a fixed point $\bar{x} \in X$; that is, $\bar{x} \in T(\bar{x})$. 
By Lemma 9, we can obtain the following collectively fixed point theorem which is the main result of our paper.

Theorem 10. Let I be a finite index set; let $\left\{\left(X_{i} ; \Gamma_{i}\right)\right\}_{i \in I}$ be a family of abstract convex spaces such that $(X ; \Gamma):=\left(\prod_{i \in I} X_{i} ; \Gamma\right)$ is an abstract convex space defined as in Lemma 7. Let $K$ be nonempty compact subset $X$. For each $i \in I$, let $S_{i}, T_{i}: X \rightarrow$ $2^{X_{i}}$ be set-valued mappings such that,

(i) for each $x \in X, \operatorname{co}_{\Gamma_{i}} S_{i}(x) \subseteq T_{i}(x)$;

(ii) for each $y_{i} \in X_{i}, S_{i}^{-1}\left(y_{i}\right)$ is open in $X$;

(iii) $K \subseteq \bigcup_{y_{i} \in X_{i}} S_{i}^{-1}\left(y_{i}\right)$;

(iv) for each $N_{i} \in\left\langle X_{i}\right\rangle$, there exists a compact $\Gamma_{i}$-convex subset $L_{N_{i}}$ of $\left(X_{i} ; \Gamma_{i}\right)$ containing $N_{i}$ such that, for $L:=$ $\prod_{i \in I} L_{N_{i}}$, we have

$$
L \backslash K \subseteq \bigcup\left\{S_{i}^{-1}\left(y_{i}\right): y_{i} \in L_{N_{i}}\right\} .
$$

If $(X ; \Gamma)$ satisfies $1_{X} \in \mathfrak{I C E}(X, X)$, then there exists $\bar{x}=$ $\left(\bar{x}_{i}\right)_{i \in I} \in X$ such that $\bar{x}_{i} \in T_{i}(\bar{x})$ for each $i \in I$.

Proof. Since $K$ is compact subset of $X$, by (ii) and (iii), for each $i \in I$, there exists $N_{i} \in\left\langle X_{i}\right\rangle$ such that

$$
K \subseteq \bigcup\left\{S_{i}^{-1}\left(y_{i}\right): y_{i} \in N_{i}\right\} .
$$

Then by (iv), for each $i \in I$, there exists a compact $\Gamma_{i^{-}}$ convex subset $L_{N_{i}}$ of $\left(X_{i} ; \Gamma_{i}\right)$ containing $N_{i}$ such that, for $L:=\prod_{i \in I} L_{N_{i}}$, we have

$$
L \backslash K \subseteq \bigcup\left\{S_{i}^{-1}\left(y_{i}\right): y_{i} \in L_{N_{i}}\right\} .
$$

By (4), we have

$$
L \bigcap K \subseteq \bigcup\left\{S_{i}^{-1}\left(y_{i}\right): y_{i} \in L_{N_{i}}\right\} .
$$

Then it follows from (5) and (6) that

$$
\begin{aligned}
L & =(L \backslash K) \bigcup(L \bigcap K) \\
& =\bigcup\left\{S_{i}^{-1}\left(y_{i}\right) \bigcap L: y_{i} \in L_{N_{i}}\right\}, \quad \forall i \in I .
\end{aligned}
$$

For each $i \in I$, since $L_{N_{i}}$ is $\Gamma_{i}$-convex subset of $\left(X_{i} ; \Gamma_{i}\right)$, it follows from Lemma 1 in Park [9] that $\left(L_{N_{i}} ;\left.\Gamma_{i}\right|_{\left\langle L_{N_{i}}\right\rangle}\right)$ is an abstract convex space, which is a subspace of $\left(X_{i} ; \Gamma_{i}\right)$. Then by Lemma $7,\left(L ;\left.\Gamma\right|_{\langle L\rangle}\right)$ is an abstract convex space, which is a subspace of $(X ; \Gamma)$. Now, for each $i \in I$, define two set-valued mappings $S_{i}^{\prime}, T_{i}^{\prime}: L \rightarrow 2^{L_{N_{i}}}$ by

$$
S_{i}^{\prime}(x)=S_{i}(x) \bigcap L_{N_{i}}, \quad T_{i}^{\prime}(x)=T_{i}(x) \bigcap L_{N_{i}}, \quad \forall x \in L .
$$

Furthermore, we define two set-valued mappings $S^{\prime}, T^{\prime}$ : $L \rightarrow 2^{L}$ by

$$
\begin{aligned}
S^{\prime}(x) & =\prod_{i \in I} S_{i}^{\prime}(x), \quad \forall x \in L, \\
T^{\prime}(x) & =\prod_{i \in I} T_{i}^{\prime}(x), \quad \forall x \in L .
\end{aligned}
$$

Next, we prove that $S^{\prime}$ and $T^{\prime}$ satisfy all the conditions of Lemma 9 as follows.

(a) For each $x \in L, M \in\left\langle S^{\prime}(x)\right\rangle$ implies that $\left.\Gamma\right|_{\langle L\rangle}(M) \subseteq$ $T^{\prime}(x)$. In fact, $M \in\left\langle S^{\prime}(x)\right\rangle$ implies that $\pi_{i}(M) \in$ $\left\langle S_{i}^{\prime}(x)\right\rangle$ for each $i \in I$. Then it follows that $\pi_{i}(M) \in$ $\left\langle S_{i}(x)\right\rangle$ for each $i \in I$. By (i) and the fact that $\left(L_{N_{i}} ;\left.\Gamma_{i}\right|_{\left\langle L_{N_{i}}\right\rangle}\right)$ is a subspace of $\left(X_{i} ; \Gamma_{i}\right)$ for each $i \in I$, we have

$$
\begin{gathered}
\Gamma_{i}\left(\pi_{i}(M)\right)=\left.\Gamma_{i}\right|_{\left\langle L_{N_{i}}\right\rangle}\left(\pi_{i}(M)\right) \subseteq T_{i}(x), \quad \forall i \in I, \\
\Gamma_{i}\left(\pi_{i}(M)\right)=\left.\Gamma_{i}\right|_{\left\langle L_{N_{i}}\right\rangle}\left(\pi_{i}(M)\right) \subseteq L_{N_{i}}, \quad \forall i \in I .
\end{gathered}
$$

Then it follows from (10) that

$$
\begin{aligned}
\left.\Gamma\right|_{\langle L\rangle}(M) & =\prod_{i \in I}\left(\left.\Gamma_{i}\right|_{\left\langle L_{N_{i}}\right\rangle}\left(\pi_{i}(M)\right)\right) \\
& \subseteq \prod_{i \in I}\left(T_{i}(x) \bigcap L_{N_{i}}\right)=T^{\prime}(x),
\end{aligned}
$$

which implies that $\operatorname{co}_{\left.\Gamma\right|_{\langle L\rangle}} S^{\prime}(x) \subseteq T^{\prime}(x)$ for each $x \in L$.

(b) For each $y \in L,\left(S^{\prime}\right)^{-1}(y)$ is relatively open in $L$. In fact, by (8), for each $i \in I$ and each $y_{i} \in L_{N_{i}}$, we have

$$
\begin{aligned}
\left(S_{i}^{\prime}\right)^{-1}\left(y_{i}\right) & =\left\{x \in L: y_{i} \in S_{i}(x) \bigcap L_{N_{i}}\right\} \\
& =L \bigcap\left\{x \in X: y_{i} \in S_{i}(x)\right\} \\
& =L \bigcap S_{i}^{-1}\left(y_{i}\right) .
\end{aligned}
$$

Therefore, for each $y \in L$, we obtain

$$
\begin{aligned}
\left(S^{\prime}\right)^{-1}(y) & =\left\{x \in L: y \in S^{\prime}(x)\right\} \\
& =\left\{x \in L: y_{i} \in S_{i}^{\prime}(x), \forall i \in I\right\} \\
& =\left\{x \in L: x \in\left(S_{i}^{\prime}\right)^{-1}\left(y_{i}\right), \forall i \in I\right\} \\
& =\bigcap_{i \in I}\left(S_{i}^{\prime}\right)^{-1}\left(y_{i}\right) \\
& =L \bigcap\left(\bigcap_{i \in I} S_{i}^{-1}\left(y_{i}\right)\right) .
\end{aligned}
$$

By (ii) and the fact that $I$ is a finite index set, we know that $\left(S^{\prime}\right)^{-1}(y)$ is relatively open in $L$ for each $y \in L$.

(c) There exists $\left\{\bar{y}_{0}, \ldots, \bar{y}_{n}\right\} \in\langle L\rangle$ such that $L=$ $\bigcup_{j=0}^{n}\left(S^{\prime}\right)^{-1}\left(\bar{y}_{j}\right)$. In fact, it follows from (7) and (12) that

$$
L=\bigcup\left\{\left(S_{i}^{\prime}\right)^{-1}\left(y_{i}\right): y_{i} \in L_{N_{i}}\right\}, \quad \forall i \in I .
$$

Let $x \in L$ be given. Then by (14), for each $i \in I$, there exists $y_{i} \in S_{i}^{\prime}(x)$ such that

$$
x \in \bigcap_{i \in I}\left(s_{i}^{\prime}\right)^{-1}\left(y_{i}\right)=\left(s^{\prime}\right)^{-1}(y), \quad \text { where } y=\left(y_{i}\right)_{i \in I} \in L \text {. }
$$


Therefore, from the arbitrary of $x$, we have that $L=\bigcup_{y \in L}\left(S^{\prime}\right)^{-1}(y)$. Since $L$ is compact, there exists $\left\{\bar{y}_{0}, \ldots, \bar{y}_{n}\right\} \in\langle L\rangle$ such that $L=\bigcup_{j=0}^{n}\left(S^{\prime}\right)^{-1}\left(\bar{y}_{j}\right)$.

(d) Since $1_{X} \in \mathfrak{\Re} \mathfrak{S}(X, X)$, it follows from Lemma 8 that $1_{L} \in \mathfrak{\Re} \mathfrak{S}(L, L)$.

Hence, by Lemma 9, there exists $\bar{x}=\left(\bar{x}_{i}\right)_{i \in I} \in L \subseteq X$ such that $x \in T^{\prime}(x)$; that is, $\bar{x}_{i} \in T_{i}^{\prime}(\bar{x}) \subseteq T_{i}(\bar{x})$ for each $i \in I$. This completes the proof.

Remark 11. Theorem 10 is a new result and completely different from the corresponding collectively fixed point theorems in [1-6], the proofs of which are mainly based on the unity partition theorem. Therefore, the topological spaces in these fixed point theorems satisfy Hausdorff property.

Theorem 12. Let I be a finite index set; let $\left\{\left(X_{i} ; \Gamma_{i}\right)\right\}_{i \in I}$ be a family of abstract convex spaces such that $(X ; \Gamma):=\left(\prod_{i \in I} X_{i} ; \Gamma\right)$ is an abstract convex space defined as in Lemma 7 . Let $K$ be nonempty compact subset $X$. For each $i \in I$, let $S_{i}, T_{i}: X \rightarrow$ $2^{X_{i}}$ be set-valued mappings such that,

(i) for each $x \in X, \operatorname{co}_{\Gamma_{i}} S_{i}(x) \subseteq T_{i}(x)$;

(ii) $K \subseteq \bigcup_{y_{i} \in X_{i}} \operatorname{int} S_{i}^{-1}\left(y_{i}\right)$;

(iii) for each $N_{i} \in\left\langle X_{i}\right\rangle$, there exists a compact $\Gamma_{i}$-convex subset $L_{N_{i}}$ of $\left(X_{i} ; \Gamma_{i}\right)$ containing $N_{i}$ such that, for $L:=$ $\prod_{i \in I} L_{N_{i}}$, we have

$$
L \backslash K \subseteq \bigcup\left\{\operatorname{int} S_{i}^{-1}\left(y_{i}\right): y_{i} \in L_{N_{i}}\right\} .
$$

If $(X ; \Gamma)$ satisfies $1_{X} \in \mathfrak{\Re} \mathfrak{C}(X, X)$, then there exists $\bar{x}=$ $\left(\bar{x}_{i}\right)_{i \in I} \in X$ such that $\bar{x}_{i} \in T_{i}(\bar{x})$ for each $i \in I$.

Proof. For each $i \in I$, define a set-valued mapping $\widetilde{S_{i}}: X \rightarrow$ $2^{X_{i}}$ as follows:

$$
\widetilde{S_{i}}(x)=\left(\operatorname{int} S_{i}^{-1}\right)^{-1}(x), \quad \forall x \in X .
$$

Then by (i), we have $\operatorname{co}_{\Gamma_{i}} \widetilde{S_{i}}(x) \subseteq T_{i}(x)$ for each $i \in I$ and each $x \in \mathrm{X}$. By (17), for each $i \in I$ and each $y_{i} \in X_{i}$, we have

$$
{\widetilde{S_{i}}}^{-1}\left(y_{i}\right)=\operatorname{int} S_{i}^{-1}\left(y_{i}\right),
$$

which is open in $X$. By (ii) and (18), we have $K \subseteq$ $\bigcup_{y_{i} \in X_{i}}{\widetilde{S_{i}}}^{-1}\left(y_{i}\right)$ for each $i \in I$. By (iii) and (18), there exists a nonempty compact subset $K$ of $X$ such that, for each $i \in I$ and each $N_{i} \in\left\langle X_{i}\right\rangle$, there exists a compact $\Gamma_{i}$-convex subset $L_{N_{i}}$ of $\left(X_{i} ; \Gamma_{i}\right)$ containing $N_{i}$ such that, for $L:=\prod_{i \in I} L_{N_{i}}$, we have

$$
L \backslash K \subseteq \bigcup\left\{{\widetilde{S_{i}}}^{-1}\left(y_{i}\right): y_{i} \in L_{N_{i}}\right\} .
$$

Since $(X ; \Gamma)$ satisfies $1_{X} \in \mathfrak{\Re} \mathfrak{C}(X, X)$, it follows that all the conditions of Theorem 10 for $\widetilde{S_{i}}$ and $T_{i}$ hold. Therefore, by Theorem 10, there exists $\bar{x}=\left(\bar{x}_{i}\right)_{i \in I} \in X$ such that $\bar{x}_{i} \in T_{i}(\bar{x})$ for each $i \in I$.

Remark 13. We have shown that Theorem 10 implies Theorem 12. It is clear that Theorem 12 implies Theorem 10. Therefore, Theorem 10 is equivalent to Theorem 12.

\section{Particular Fixed Point Theorems}

In this section, we give simple consequences of Theorems 10 and 12 and their applications obtained by other authors. We omit their proofs.

Proposition 14 (see [21]). Let $X_{1}, \ldots, X_{n}(n \geq 1)$ be nonempty compact convex sets, each in a topological vector space (not necessary Hausdorff). Let each $M_{i}: X \rightarrow 2^{X_{i}}$ have the local intersection property and nonempty convex values. Then there exists $\bar{x} \in X$ such that $\bar{x}_{i} \in M_{i}(\bar{x})$ for each $i \in\{1, \ldots, n\}$.

Remark 15. By Remarks 6 and 13, we know that Proposition 14 is a particular form of Theorems 10 and 12 .

By using Proposition 14, Prokopovych [21] proved a theorem on the existence of a pure strategy $\varepsilon$-Nash equilibrium in every compact, quasiconcave, and payoff secure game; meanwhile, by means of Proposition 14, he also proved an approximate equilibrium existence theorem that covers a number of known game models.

By Proposition 14, we can obtain the following famous Fan-Browder fixed theorem.

The Fan-Browder Fixed Point Theorem (see [22, 23]). Let $X$ be a nonempty compact convex subset of a topological vector space. Let $M: X \rightarrow 2^{X}$ be a set-valued mapping such that it has nonempty convex values and open inverse values (i.e., each $M^{-1}(y)$ is open in $\left.X\right)$. Then $M$ has a fixed point.

By using the Fan-Browder fixed point theorem, Yu and Yuan [24] obtained the existence results of weight Nashequilibria and Pareto equilibria for multiobjective games. Kim and Yuan [25] applied the Fan-Browder fixed point theorem to prove a maximal element theorem for $L$-majorized mappings in topological vector spaces from which they obtained an existence theorem of maximal elements for the family of $L$-majorized mappings in which domains are not compact. Balaj and Muresan [26] obtained two minimax inequalities by using the Fan-Browder fixed point theorem. Recently, Luo [27] applied the Fan-Browder fixed point theorem to establish some generalized Ky Fan minimax inequalities for vector-valued mappings.

In 1992, Park [28] generalized the Fan-Browder fixed point theorem to noncompact setting and obtained the following result, which is a particular case of Theorems 10 and 12 with $I=\{1\}$.

Proposition 16 (see [28]). Let $X$ be a nonempty convex subset of a topological vector space, $A \subseteq X$ nonempty convex, and $D \subseteq A$ a nonempty compact subset. Let $S: A \rightarrow 2^{A}$ and $L: A \rightarrow 2^{A}$ be two set-valued mappings. Assume that,

(a) for each $x \in A, L(x)$ is convex and $S(x) \subseteq L(x)$;

(b) for each $x \in D, S(x) \neq \emptyset$;

(c) for each $y \in A, S^{-1}(y)$ is open in $A$;

(d) for each finite subset $N$ of $A$, there is a compact convex 
subset $L_{N}$ such that $N \subseteq L_{N} \subseteq A$ and $S(x) \cap L_{N} \neq \emptyset$ for all $x \in L_{N} \backslash D$.

Then $L$ has a fixed point.

Remark 17. The coercivity condition (d) in Proposition 16 can be replaced by the following condition:

(d)' for each finite subset $N$ of $A$, there is a compact convex subset $L_{N}$ such that $N \subseteq L_{N} \subseteq A$ and $L_{N} \backslash D \subseteq$ $\bigcup_{y \in L_{N}} S^{-1}(y)$.

Many authors applied Proposition 16 or particular forms of Proposition 16 to study various nonlinear problems in topological vector spaces, for example, generalized Minty vector variational inequality problems, generalized variational inequality problems, generalized vector equilibrium problems, and the constrained or the competitive Nash type equilibrium problems. For more details, see Park [29] and the references therein.

The following particular form of Proposition 16 can be found in Ding and Tan [30].

Proposition 18 (see [30]). Let $X$ be a nonempty convex subset of a topological vector space. Let $S: X \rightarrow 2^{X}$ be a set-valued mapping. Assume that,

(a) for each $x \in X, S(x) \neq \emptyset$;

(b) for each $y \in X, S^{-1}(y)$ is compactly open; that is, for each nonempty compact subset $C$ of $X, S^{-1}(y) \cap C$ is open in C;

(c) there exist a nonempty compact convex subset $X_{0}$ of $X$ and a nonempty compact subset $K$ of $X$ (not necessarily convex) such that, for each $x \in X \backslash K$, we have $\operatorname{co}\left(X_{0} \bigcup\{x\}\right) \bigcap \cos (x) \neq \emptyset$.

Then there exists $\bar{x}$ such that $\bar{x} \in \operatorname{coS}(\bar{x})$.

Ding and Tan [30] applied Proposition 18 to obtain an existence theorem of equilibria for one person games. By using Proposition 18, Ding and Yuan [31] proved a maximal element theorem from which they obtained some existence theorems of equilibria for generalized games without lower semicontinuity for both constraint and preference correspondences.

Remark 19. In condition (b) of Proposition 18, "compactly" can be removed; see Park [11].

The following result (see Corollary 2.3 in Tarafdar [2]) is known in the setting of $\mathrm{H}$-spaces without linear structure.

Proposition 20 (see [2]). Let $\left(X, F_{A}\right)$ be a compact $H$-space and $T: X \rightarrow 2^{X}$ a set-valued mapping such that,

(a) for each $x \in X, T(x)$ is a nonempty $H$-convex subset of $X$

(b) for each $y \in X, T^{-1}(y)$ contains an open set $O_{y}\left(O_{y}\right.$ may be empty for some $y$ );

(c) $X=\bigcup_{y \in X} O_{y}$.

Then $\mathrm{T}$ has a fixed point.
By using Proposition 20, Chang et al. [32] proved an existence theorem of solutions for the quasi-variational inequality problem in the setting of $H$-spaces. After that, Chang et al. [33] applied Proposition 20 with each $T^{-1}(y)$ being open to prove some existence theorems of loose saddle point, saddle point, and minimax problems for vector-valued multifunctions in the framework of $H$-spaces. On the basis of an equivalent form of Proposition 20, Wu [34] obtained two existence theorems for maximal elements in $H$-spaces from which he proved the existence of solutions of FanYen minimax inequalities, qualitative games, and abstract economies.

The following result is a noncompact generalization of Proposition 20.

Proposition 21 (see [35]). Let $\left(X, F_{A}\right)$ be an $H$-space, $D \subseteq X$ an $H$-compact set, and $T: X \rightarrow 2^{X}$ a set-valued mapping such that,

(a) for each $x \in X, T(x)$ is a nonempty $H$-convex subset of $X$;

(b) for each $y \in X, T^{-1}(y)$ is open in $X$;

(c) $T(x) \cap D \neq \emptyset$ for all $x \in X$.

Then $T$ has a fixed point.

By using Proposition 21, Cubiotti and Nordo [36] obtained an existence result for the Nash equilibria of generalized games with strategy sets in $H$-spaces.

Luo [37] proved the following fixed point theorem in topological ordered spaces.

Proposition 22 (see [37]). Let $X$ be a nonempty compact $\Delta$ convex subset of a topological semilattice with path-connected intervals and $T: X \rightarrow 2^{X}$ a set-valued mapping such that

(a) for each $x \in X, T(x)$ is nonempty and $\Delta$-convex;

(b) for each $y \in X, T^{-1}(y)$ is open in $X$.

Then $T$ has a fixed point.

Luo $[37,38]$ applied Proposition 22 to prove a saddlepoint theorem, existence theorems of solutions for some generalized quasi-Ky Fan inequalities, and Nash equilibrium points for a game system in the setting of topological ordered spaces. By using Proposition 22, Vinh [39] proved a coincidence theorem from which he obtained a Sion-Neumann type minimax theorem.

As it is well known, $G$-convex spaces are typical example of abstract convex spaces. The following extension of the Fan-Browder fixed point theorem to $G$-convex spaces is a particular form of Theorem 3.3 in Park [29], and it includes the fixed point theorems mentioned previously as special cases.

Proposition 23. Let $(X ; \Gamma)$ be a G-convex space, and let $S, T$ : $X \rightarrow 2^{X}$ be two set-valued mappings such that,

(a) for each $x \in X, N \in\langle S(x)\rangle$ implies $\Gamma(A) \subseteq T(x)$;

(b) $X=\bigcup_{y \in X} \operatorname{int} S^{-1}(y)$; 
(c) there exists a nonempty compact subset $K$ of $X$ such that, for each $N \in\langle X\rangle$, there exists a compact $G$ convex subset $L_{N}$ of $(X ; \Gamma)$ containing $N$ such that

$$
L \backslash K \subseteq \bigcup\left\{\operatorname{int} S^{-1}(y): y \in L_{N}\right\} .
$$

Then there exists $\bar{x} \in X$ such that $\bar{x} \in T(\bar{x})$.

Remark 24. The coercivity condition (c) in Proposition 23 can be replaced by the following equivalent condition:

(c) ${ }^{\prime}$ there exists a nonempty compact subset $K$ of $X$ such that, for each $N \in\langle X\rangle$, there exists a compact $G$ convex subset $L_{N}$ of $(X ; \Gamma)$ containing $N$ such that

$$
L \bigcap\left(\bigcap\left\{X \backslash \operatorname{int} S^{-1}(y): y \in L_{N}\right\}\right) \subseteq K \text {. }
$$

Lin [19] applied the equivalent form of Proposition 23 to obtain some minimax inequalities, existence of maximal element, intersection theorems, and KKM type theorems. At the same year, Lin and Yu [40] applied special cases of Proposition 23 to study scalar equilibrium problems and vectorial equilibrium problems in the setting of $G$-convex spaces. Ding and Park [41] applied Proposition 23 to a class of abstract generalized vector equilibrium problems in $G$ convex spaces. Recently, by using Proposition 23, Balaj and Lin [42] proved a new fixed point theorem for set-valued mappings in $G$-convex spaces from which they obtained some coincidence theorems and existence theorems for maximal elements. Applications of these results to generalized equilibrium and minimax theory were also given.

In 2010, Park [7] established the following generalized Fan-Browder fixed point theorem in abstract convex spaces.

Proposition 25 (see [7]). Let I be a finite index set; let $\left\{\left(X_{i} ; \Gamma_{i}\right)\right\}_{i \in I}$ be a family of compact abstract convex spaces such that $(X ; \Gamma):=\left(\prod_{i \in I} X_{i} ; \Gamma\right)$ is an abstract convex space as defined as in Lemma 7, and satisfies the partial KKM principle. For each $i \in I$, let $T_{i}: X \rightarrow 2^{X_{i}}$ be a set-valued mapping such that,

(a) for each $x \in X, \operatorname{co}_{\Gamma_{i}} S_{i}(x) \subseteq T_{i}(x)$;

(b) $X=\bigcup_{y_{i} \in X_{i}} \operatorname{int} S_{i}^{-1}\left(y_{i}\right)$.

Then there exists $\bar{x}=\left(\bar{x}_{i}\right)_{i \in I} \in X$ such that $\bar{x}_{i} \in T_{i}(\bar{x})$ for each $i \in I$.

Park [7] applied Proposition 25 to establish the von Neumann-Fan type intersection theorem under the setting of abstract convex spaces satisfying the partial KKM principle. By using Proposition 25 with $I$ being a singleton and $S=$ $T$, Yang et al. [43] established some minimax theorems for vector-valued mappings in abstract convex spaces. They also gave some examples to illustrate their results.

\section{Equilibria for Generalized Abstract Economies}

Considering any preference of a real agent could be unstable because of the fuzziness of consumers' behavior or market situations, Kim and Tan [44] introduced the fuzzy constraint correspondences in defining the following generalized abstract economy.

Let $I$ be any set of agents. For each $i \in I$, let $X_{i}$ be the strategy set or commodity space of the agent $i$, and let $X=\prod_{i \in I} X_{i}$. Following the method of Kim and Tan [44], let $\varepsilon=\left(X_{i}, A_{i}, B_{i}, F_{\mathrm{i}}, P_{i}\right)_{i \in I}$ be a generalized abstract economy, where $A_{i}, B_{i}: X=\prod_{i \in I} X_{i} \rightarrow 2^{X_{i}}$ are two constrained correspondences such that $A_{i}(x)$ and $B_{i}(x)$ are the states attainable for the agent $i$ at $x ; F_{i}: X \rightarrow 2^{X_{i}}$ is a fuzzy constrained correspondence such that $F_{i}(x)$ is the unstable state for the agent $i$ at $x$, and $P_{i}: X \times X \rightarrow 2^{X_{i}}$ is a preference correspondence such that $P_{i}(x, y)$ is the state preference by the agent $i$ at $(x, y)$. An equilibrium for $\varepsilon$ is a point $(\bar{x}, \bar{y}) \epsilon$ $X \times X$ such that for each $i \in I, \bar{x}_{i} \in B_{i}(\bar{x}), \bar{y}_{i} \in F_{i}(\bar{x})$, and $P_{i}(\bar{x}, \bar{y}) \cap A_{i}(\bar{x})=\emptyset$.

As an application of Theorem 10, we derive the following equilibrium existence theorem for generalized abstract economies in noncompact abstract convex spaces.

Theorem 26. Let I be a finite index set, $\left\{\left(X_{i} ; \Gamma_{i}\right)\right\}_{i \in I}$ a family of abstract convex spaces such that $(X ; \Gamma):=\left(\prod_{i \in I} X_{i} ; \Gamma\right)$ and $(X \times X ; \Gamma \times \Gamma)$ are two abstract convex spaces as defined in Lemma 7. Let $\varepsilon=\left(\left(X_{i} ; \Gamma_{i}\right), A_{i}, B_{i}, F_{i}, P_{i}\right)_{i \in I}$ be a generalized abstract economy, and let $K$ be a nonempty compact subset of $X \times X$. For each $i \in I$, assume that,

(i) for each $x \in X, A_{i}(x) \neq \emptyset$ and $\operatorname{co}_{\Gamma_{i}} A_{i}(x) \subseteq B_{i}(x)$;

(ii) for each $x \in X, F_{i}(x)$ is nonempty $\Gamma_{i}$-convex;

(iii) for each $(x, y) \in W_{i}=\{(x, y) \in X \times X$ : $\left.A_{i}(x) \bigcap P_{i}(x, y) \neq \emptyset\right\}, x_{i} \notin \operatorname{co}_{\Gamma_{i}} P_{i}(x, y) ;$

(iv) for each $\left(u_{i}, v_{i}\right) \in X_{i} \times X_{i},\left[\left(A_{i}^{-1}\left(u_{i}\right) \bigcap F_{i}^{-1}\left(v_{i}\right)\right) \times\right.$ $X] \bigcap\left[P_{i}^{-1}\left(u_{i}\right) \bigcup\left(X \times X \backslash W_{i}\right)\right]$ is open in $X \times X$;

(v) for each $N_{0 i} \times N_{1 i} \in\left\langle X_{i} \times X_{i}\right\rangle$, there exist compact $\Gamma_{i}$ convex subsets $L_{N_{0 i}}, L_{N_{1 i}}$ of $\left(X_{i} ; \Gamma_{i}\right)$ containing $N_{0 i}, N_{1 i}$, respectively, such that, for $L:=\prod_{i \in I} L_{N_{0 i}} \times \prod_{i \in I} L_{N_{1 i}}$, we have

$$
\begin{aligned}
L \backslash K \subseteq \bigcup_{\left(u_{i}, v_{i}\right) \in L_{N_{0 i}} \times L_{N_{1 i}}}\{ & {\left[\left(A_{i}^{-1}\left(u_{i}\right) \bigcap F_{i}^{-1}\left(v_{i}\right)\right) \times X\right] } \\
& \left.\bigcap\left[P_{i}^{-1}\left(u_{i}\right) \bigcup\left(X \times X \backslash W_{i}\right)\right]\right\} .
\end{aligned}
$$

If $(X \times X ; \Gamma \times \Gamma)$ satisfies $1_{X \times X} \in \mathfrak{\Re} \mathfrak{C}(X \times X, X \times X)$, then there exists $(\bar{x}, \bar{y}) \in X \times X$ such that, for each $i \in I, \bar{x}_{i} \in B_{i}(\bar{x})$, $\bar{y}_{i} \in F_{i}(\bar{x})$ and $P_{i}(\bar{x}, \bar{y}) \cap A_{i}(\bar{x})=\emptyset$.

Proof. By Lemma 7, for each $i \in I,\left(X_{i} \times X_{i} ; \Gamma_{i} \times \Gamma_{i}\right)$ is an abstract convex space. For each $i \in I$, define two set-valued mappings $S_{i}, T_{i}: X \times X \rightarrow 2^{\mathrm{X}_{i} \times X_{i}}$ by

$$
\begin{gathered}
S_{i}(x, y)= \begin{cases}{\left[P_{i}(x, y) \bigcap A_{i}(x)\right] \times F_{i}(x),} & \text { if }(x, y) \in W_{i}, \\
A_{i}(x) \times F_{i}(x), & \text { if }(x, y) \in X \times X \backslash W_{i},\end{cases} \\
T_{i}(x, y)= \begin{cases}{\left[\operatorname{co}_{\Gamma_{i}} P_{i}(x, y) \bigcap B_{i}(x)\right] \times F_{i}(x),} & \text { if }(x, y) \in W_{i}, \\
B_{i}(x) \times F_{i}(x), & \text { if }(x, y) \in X \times X \backslash W_{i} .\end{cases}
\end{gathered}
$$


By (i), (ii), and the definition of $W_{i}$, we have $S_{i}(x, y) \neq \emptyset$ and $\operatorname{CO}_{\Gamma_{i} \times \Gamma_{i}}\left(S_{i}(x, y)\right) \subseteq T_{i}(x, y)$ for each $i \in I$ and for all $(x, y) \in$ $X \times X$. For each $i \in I$ and for all $\left(u_{i}, v_{i}\right) \in X_{i} \times X_{i}$, we see from

$$
\begin{aligned}
S_{i}^{-1}\left(u_{i}, v_{i}\right)= & {\left[P_{i}^{-1}\left(u_{i}\right) \bigcap\left(A_{i}^{-1}\left(u_{i}\right) \times X\right) \bigcap\left(F_{i}^{-1}\left(v_{i}\right) \times X\right)\right] } \\
& \bigcup\left[\left(X \times X \backslash W_{i}\right) \bigcap\left(A_{i}^{-1}\left(u_{i}\right) \times X\right)\right. \\
& \left.\bigcap\left(F_{i}^{-1}\left(v_{i}\right) \times X\right)\right] \\
= & {\left[\left(A_{i}^{-1}\left(u_{i}\right) \bigcap F_{i}^{-1}\left(v_{i}\right)\right) \times X\right] } \\
& \bigcap\left[P_{i}^{-1}\left(u_{i}\right) \bigcup\left(X \times X \backslash W_{i}\right)\right]
\end{aligned}
$$

and (iv) that $S_{i}^{-1}\left(u_{i}, v_{i}\right)$ is open in $X \times X$. Since $S_{i}(x, y) \neq \emptyset$ for each $i \in I$ and for all $(x, y) \in X \times X$, we have the following:

$$
X \times X=\bigcup_{\left(u_{i}, v_{i}\right) \in X_{i} \times X_{i}} S_{i}^{-1}\left(u_{i}, v_{i}\right), \quad \forall i \in I,
$$

and so, we have

$$
K \subseteq X \times X=\bigcup_{\left(u_{i}, v_{i}\right) \in X_{i} \times X_{i}} S_{i}^{-1}\left(u_{i}, v_{i}\right), \quad \forall i \in I .
$$

By (v), for each $i \in I$ and each $N_{i}=N_{0 i} \times N_{1 i} \in\left\langle X_{i} \times X_{i}\right\rangle$, there exists a compact $\Gamma_{i} \times \Gamma_{i}$-convex subset $L_{N_{0 i}} \times L_{N_{1 i}}$ of $\left(X_{i} \times X_{i} ; \Gamma_{i} \times \Gamma_{i}\right)$ containing $N_{i}$, such that, for $L:=\prod_{i \in I} L_{N_{0 i}} \times$ $\prod_{i \in I} L_{N_{1 i}}$, we have

$$
L \backslash K \subseteq \bigcup\left\{S_{i}^{-1}\left(u_{i}, v_{i}\right):\left(u_{i}, v_{i}\right) \in L_{N_{0 i}} \times L_{N_{1 i}}\right\} .
$$

Since $(X \times X ; \Gamma \times \Gamma)$ satisfies $1_{X \times X} \in \mathfrak{I} \mathfrak{C}(X \times X, X \times X)$, we can see that all the conditions of Theorem 10 are satisfied. So, by Theorem 10 , there exists $(\bar{x}, \bar{y}) \in X \times X$ such that $\left(\bar{x}_{i}, \bar{y}_{i}\right) \in$ $T_{i}(\bar{x}, \bar{y})$ for each $i \in I$. If $(\bar{x}, \bar{y}) \in W_{i}$ for some $i$, then we have

$$
\left(\bar{x}_{i}, \bar{y}_{i}\right) \in\left[\operatorname{co}_{\Gamma_{i}} P_{i}(\bar{x}, \bar{y}) \bigcap B_{i}(\bar{x})\right] \times F_{i}(\bar{x}) .
$$

And, hence, $\bar{x}_{i} \in \operatorname{co}_{\Gamma_{i}} P_{\mathrm{i}}(\bar{x}, \bar{y}) \cap B_{i}(\bar{x})$ and so $\bar{x}_{i} \in \operatorname{co}_{\Gamma_{i}} P_{i}(\bar{x}, \bar{y})$, which contradicts (iii). Therefore, we must have $(\bar{x}, \bar{y}) \in X \times$ $X \backslash W_{i}$ for all $i \in I$. It follows from the definitions of $T_{i}$ and $W_{i}$ that, for each $i \in I, \bar{x}_{i} \in B_{i}(\bar{x}), \bar{y}_{i} \in F_{i}(\bar{x})$, and $P_{i}(\bar{x}, \bar{y}) \cap$ $A_{i}(\bar{x})=\emptyset$.

Remark 27. Theorem 26 is a new result, which can be compared with Theorem 3.2.1 in [45], Theorems 4.1-4.2 in [46], and Theorem 3.1 in [47] in several aspects.

Corollary 28. Let I be a finite index set; let $\left\{\left(X_{i} ; \Gamma_{i}\right)\right\}_{i \in I}$ be a family of abstract convex spaces such that $(X ; \Gamma):=\left(\prod_{i \in I} X_{i} ; \Gamma\right)$ and $(X \times X ; \Gamma \times \Gamma)$ are two abstract convex spaces as defined in Lemma 7. Let $\varepsilon=\left(\left(X_{i} ; \Gamma_{i}\right), A_{i}, B_{i}, F_{i}, P_{i}\right)_{i \in I}$ be a generalized abstract economy, and let $K$ be a nonempty compact subset of $X \times X$. For each $i \in I$, assume that,

(i) for each $x \in X, A_{i}(x) \neq \emptyset$ and $\operatorname{co}_{\Gamma_{i}} A_{i}(x) \subseteq B_{i}(x)$;

(ii) for each $x \in X, F_{i}(x)$ is nonempty $\Gamma_{i}$-convex;

(iii) for each $(x, y) \in W_{i}=\{(x, y) \in X \times X$ : $\left.A_{i}(x) \bigcap P_{i}(x, y) \neq \emptyset\right\}, x_{i} \notin \operatorname{co}_{\Gamma_{i}} P_{i}(x, y)$; (iv) for each $u_{i} \in X_{i}, A_{i}^{-1}\left(u_{i}\right), F_{i}^{-1}\left(u_{i}\right)$, and $P_{i}^{-1}\left(u_{i}\right)$ are open sets;

(v) the set $W_{i}$ is closed in $X \times X$;

(vi) for each $N_{0 i} \times N_{1 i} \in\left\langle X_{i} \times X_{i}\right\rangle$, there exist compact $\Gamma_{i}$-convex subsets $L_{N_{0 i}}, L_{N_{1 i}}$ of $\left(X_{i} ; \Gamma_{i}\right)$ containing $N_{0 i}, N_{1 i}$, respectively, such that, for $L:=\prod_{i \in I} L_{N_{0 i}} \times$ $\prod_{i \in I} L_{N_{1 i}}$, we have

$$
\begin{aligned}
L \backslash K \subseteq \underset{\left(u_{i}, v_{i}\right) \in L_{N_{0 i}} \times L_{N_{1 i}}}{\bigcup}\{ & {\left[\left(A_{i}^{-1}\left(u_{i}\right) \bigcap F_{i}^{-1}\left(v_{i}\right)\right) \times X\right] } \\
& \left.\bigcap\left[P_{i}^{-1}\left(u_{i}\right) \bigcup\left(X \times X \backslash W_{i}\right)\right]\right\} .
\end{aligned}
$$

If $(X \times X ; \Gamma \times \Gamma)$ satisfies $1_{X \times X} \in \mathfrak{\Re} \mathfrak{S}(X \times X, X \times X)$, then there exists $(\bar{x}, \bar{y}) \in X \times X$ such that, for each $i \in I, \bar{x}_{i} \in B_{i}(\bar{x})$, $\bar{y}_{i} \in F_{i}(\bar{x})$, and $P_{i}(\bar{x}, \bar{y}) \cap A_{i}(\bar{x})=\emptyset$.

Proof. By (iv) and (v), for each $\left(u_{i}, v_{i}\right) \in X_{i} \times X_{i}$, the set $\left[\left(A_{i}^{-1}\left(u_{i}\right) \cap F_{i}^{-1}\left(v_{i}\right)\right) \times X\right] \cap\left[P_{i}^{-1}\left(u_{i}\right) \cup\left(X \times X \backslash W_{i}\right)\right]$ is open in $X \times X$. Hence, the conclusion of Corollary 28 follows from Theorem 26.

Corollary 29. Let I be a finite index set; let $\left\{\left(X_{i} ; \Gamma_{i}\right)\right\}_{i \in I}$ be a family of abstract convex spaces such that $(X ; \Gamma):=\left(\prod_{i \in I} X_{i} ; \Gamma\right)$ and $(X \times X ; \Gamma \times \Gamma)$ are two abstract convex spaces as defined in Lemma 7. Let $\varepsilon=\left(\left(X_{i} ; \Gamma_{i}\right), A_{i}, B_{i}, F_{i}, P_{i}\right)_{i \in I}$ be a generalized abstract economy, and let $K$ be a nonempty compact subset of $X \times X$. For each $i \in I$, assume that,

(i) for each $x \in X, A_{i}(x) \neq \emptyset$ and $\operatorname{co}_{\Gamma_{i}} A_{i}(x) \subseteq B_{i}(x)$;

(ii) for each $x \in X, F_{i}(x)$ is nonempty $\Gamma_{i}$-convex;

(iii) for each $(x, y) \in W_{i}=\left\{(x, y) \in X \times X: A_{i}(x) \cap\right.$ $\left.P_{i}(x, y) \neq \emptyset\right\}, x_{i} \notin \mathrm{cl}\left(\operatorname{co}_{\Gamma_{i}} P_{i}(x, y)\right)$;

(iv) for each $u_{i} \in X_{i}, A_{i}^{-1}\left(u_{i}\right), F_{i}^{-1}\left(u_{i}\right)$, and $P_{i}^{-1}\left(u_{i}\right)$ are open sets;

(v) the set $W_{i}$ is closed in $X \times X$;

(vi) for each $N_{0 i} \times N_{1 i} \in\left\langle X_{i} \times X_{i}\right\rangle$, there exist compact $\Gamma_{i}$-convex subsets $L_{N_{0 i}}, L_{N_{1 i}}$ of $\left(X_{i} ; \Gamma_{i}\right)$ containing $N_{0 i}, N_{1 i}$, respectively, such that, for $L:=\prod_{i \in I} L_{N_{0 i}} \times$ $\prod_{i \in I} L_{N_{1 i}}$, we have

$$
\begin{array}{r}
L \backslash K \subseteq \bigcup_{\left(u_{i}, v_{i}\right) \in L_{N_{0 i}} \times L_{N_{1 i}}}\left\{\left[\left(A_{i}^{-1}\left(u_{i}\right) \bigcap F_{i}^{-1}\left(v_{i}\right)\right) \times X\right]\right. \\
\\
\left.\bigcap\left[P_{i}^{-1}\left(u_{i}\right) \bigcup\left(X \times X \backslash W_{i}\right)\right]\right\} .
\end{array}
$$

If $(X \times X ; \Gamma \times \Gamma)$ satisfies $1_{X \times X} \in \mathfrak{\Re} \mathfrak{C}(X \times X, X \times X)$, then there exists $(\bar{x}, \bar{y}) \in X \times X$ such that, for each $i \in I, \bar{x}_{i} \in B_{i}(\bar{x})$, $\bar{y}_{i} \in F_{i}(\bar{x})$, and $P_{i}(\bar{x}, \bar{y}) \cap A_{i}(\bar{x})=\emptyset$.

Proof. By (iii), for each $(x, y) \in W_{i}=\{(x, y) \in X \times X$ : $\left.A_{i}(x) \cap P_{i}(x, y) \neq \emptyset\right\}, x_{i} \notin \operatorname{co}_{\Gamma_{i}} P_{i}(x, y)$. Hence, the conclusion of Corollary 29 follows from Corollary 28. 
If $A_{i}(x)=B_{i}(x)=X_{i}$ for each $i \in I$ and each $x \in X$ in Theorem 26, then we have the following theorem.

Theorem 30. Let I be a finite index set; let $\left\{\left(X_{i} ; \Gamma_{i}\right)\right\}_{i \in I}$ be a family of abstract convex spaces such that $(X ; \Gamma):=\left(\prod_{i \in I} X_{i} ; \Gamma\right)$ and $(X \times X ; \Gamma \times \Gamma)$ are two abstract convex spaces as defined as in Lemma 7. Let $\varepsilon=\left(\left(X_{i} ; \Gamma_{i}\right), F_{i}, P_{i}\right)_{i \in I}$ be a generalized qualitative game, and let $K$ be a nonempty compact subset of $X \times X$. For each $i \in I$, assume that,

(i) for each $(x, y) \in W_{i}=\left\{(x, y) \in X \times X: P_{i}(x, y) \neq \emptyset\right\}$, $x_{i} \notin \operatorname{co}_{\Gamma_{i}} P_{i}(x, y)$;

(ii) for each $\left(u_{i}, v_{i}\right) \in X_{i} \times X_{i},\left[F_{i}^{-1}\left(v_{i}\right) \times X\right]$ $\bigcap\left[P_{i}^{-1}\left(u_{i}\right) \cup\left(X \times X \backslash W_{i}\right)\right]$ is open in $X \times X$;

(iii) for each $N_{0 i} \times N_{1 i} \in\left\langle X_{i} \times X_{i}\right\rangle$, there exist compact $\Gamma_{i}$-convex subsets $L_{N_{0 i}}, L_{N_{1 i}}$ of $\left(X_{i} ; \Gamma_{i}\right)$ containing $N_{0 i}, N_{1 i}$, respectively, such that, for $L:=\prod_{i \in I} L_{N_{0 i}} \times$ $\prod_{i \in I} L_{N_{1 i}}$, we have

$$
\begin{aligned}
L \backslash K \subseteq \bigcup_{\left(u_{i}, v_{i}\right) \in L_{N_{0 i}} \times L_{N_{1 i}}}\{ & {\left[F_{i}^{-1}\left(v_{i}\right) \times X\right] } \\
& \left.\bigcap\left[P_{i}^{-1}\left(u_{i}\right) \bigcup\left(X \times X \backslash W_{i}\right)\right]\right\} .
\end{aligned}
$$

If $(X \times X ; \Gamma \times \Gamma)$ satisfies $1_{X \times X} \in \mathfrak{\Omega} \mathfrak{C}(X \times X, X \times X)$, then the generalized qualitative game $\varepsilon$ has a constrained maximal element; that is, there exists $(\bar{x}, \bar{y}) \in X \times X$ such that, for each $i \in I, \bar{y}_{i} \in F_{i}(\bar{x})$ and $P_{i}(\bar{x}, \bar{y})=\emptyset$.

By Theorem 30, we can obtain the following equilibrium existence theorem for generalized abstract economies.

Theorem 31. Let I be a finite index set; let $\left\{\left(X_{i} ; \Gamma_{i}\right)\right\}_{i \in I}$ be a family of abstract convex spaces such that $(X ; \Gamma):=\left(\prod_{i \in I} X_{i} ; \Gamma\right)$ and $(X \times X ; \Gamma \times \Gamma)$ are two abstract convex spaces as defined in Lemma 7. Let $\varepsilon=\left(\left(X_{i} ; \Gamma_{i}\right), A_{i}, B_{i}, F_{i}, P_{i}\right)_{i \in I}$ be a generalized abstract economy, and let $K$ be a nonempty compact subset of $X \times X$. For each $i \in I$, assume that,

(i) the set $V_{i}=\left\{x \in X: x_{i} \notin B_{i}(x)\right\}$ is open in $X$;

(ii) if $(x, y) \in X \times X$ with $x \in V_{i}$, we have $A_{i}(x) \neq \emptyset$ and $x_{i} \notin \mathrm{Co}_{\Gamma_{i}} A_{i}(x)$; if $(x, y) \in X \times X$ with $x \notin V_{i}$ and $P_{i}(x, y) \cap A_{i}(x) \neq \emptyset$, we have $x_{i} \notin \mathrm{Co}_{\Gamma_{i}}\left(P_{i}(x, y) \cap A_{i}(x)\right)$;

(iii) for each $u_{i} \in X_{i}, A_{i}^{-1}\left(u_{i}\right), F_{i}^{-1}\left(u_{i}\right)$, and $P_{i}^{-1}\left(u_{i}\right)$ are open sets;

(iv) the set $W_{i}=\left\{(x, y) \in X \times X: x \in V_{i}\right.$ and $\left.A_{i}(x) \neq \emptyset\right\} \bigcup\left\{(x, y) \in X \times X: x \notin V_{i}\right.$ and $\left.P_{i}(x, y) \cap A_{i}(x) \neq \emptyset\right\}$ is closed in $X \times X$;

(v) for each $N_{0 i} \times N_{1 i} \in\left\langle X_{i} \times X_{i}\right\rangle$, there exist compact $\Gamma_{i}$-convex subsets $L_{N_{0 i}}, L_{N_{1 i}}$ of $\left(X_{i} ; \Gamma_{i}\right)$ containing
$N_{0 i}, N_{1 i}$, respectively, such that, for each $(x, y) \in L \backslash K$, there exists $\left(u_{i}, v_{i}\right) \in N_{0 i} \times N_{1 i}$ satisfying

$$
\begin{gathered}
(x, y) \in F_{i}^{-1}\left(v_{i}\right) \times X \\
(x, y) \in\left(\left(P_{i}^{-1}\left(u_{i}\right) \bigcup\left(V_{i} \times X\right)\right) \bigcap\left(A_{i}^{-1}\left(u_{i}\right) \times X\right)\right) \\
\bigcup\left(X \times X \backslash W_{i}\right),
\end{gathered}
$$

where $L:=\prod_{i \in I} L_{N_{0 i}} \times \prod_{i \in I} L_{N_{1 i}}$.

If $(X \times X ; \Gamma \times \Gamma)$ satisfies $1_{X \times X} \in \mathfrak{\Re} \mathfrak{S}(X \times X, X \times X)$, then there exists $(\bar{x}, \bar{y}) \in X \times X$ such that, for each $i \in I, \bar{x}_{i} \in B_{i}(\bar{x})$, $\bar{y}_{i} \in F_{i}(\bar{x})$, and $P_{i}(\bar{x}, \bar{y}) \cap A_{i}(\bar{x})=\emptyset$.

Proof. For each $i \in I$, define a set-valued mapping $H_{i}: X \times$ $X \rightarrow 2^{X_{i}}$ by

$$
H_{i}(x, y)= \begin{cases}P_{i}(x, y) \bigcap A_{i}(x), & \text { if }(x, y) \in X \times X \text { with } x \notin V_{i}, \\ A_{i}(x), & \text { if }(x, y) \in X \times X \text { with } x \in V_{i}\end{cases}
$$

Then for each $i \in I$ and each $u_{i} \in X_{i}$, we have

$$
\begin{aligned}
& H_{i}^{-1}\left(u_{i}\right)=\left\{(x, y) \in X \times X: u_{i} \in H_{i}(x, y)\right\} \\
&=\left\{(x, y) \in X \times X: x \notin V_{i}\right. \\
&\text { and } \left.u_{i} \in P_{i}(x, y) \bigcap A_{i}(x)\right\} \\
& \\
& \bigcup\left\{(x, y) \in X \times X: x \in V_{i} \text { and } u_{i} \in A_{i}(x)\right\} \\
&=\left[P_{i}^{-1}\left(u_{i}\right) \bigcap\left(\left(A_{i}^{-1}\left(u_{i}\right) \bigcap\left(X \backslash V_{i}\right)\right) \times X\right)\right] \\
& \bigcup\left[\left(V_{i} \bigcap A_{i}^{-1}\left(u_{i}\right)\right) \times X\right] \\
&= {\left[P_{i}^{-1}\left(u_{i}\right) \bigcup\left(V_{i} \times X\right)\right] \bigcap\left[A_{i}^{-1}\left(u_{i}\right) \times X\right] . }
\end{aligned}
$$

By (i), (iii), and (iv), for each $i \in I$ and each $\left(u_{i}, v_{i}\right) \in X_{i} \times X_{i}$, the set $\left[F_{i}^{-1}\left(v_{i}\right) \times X\right] \cap\left[H_{i}^{-1}\left(u_{i}\right) \cup\left(X \times X \backslash W_{i}\right)\right]$ is open in $X \times X$. By (ii), for each $i \in I$ and each $(x, y) \in X \times X$ with $H_{i}(x, y) \neq \emptyset$, we have $x_{i} \notin \operatorname{co}_{\Gamma_{i}} H_{i}(x, y)$. By (v), for each $i \in I$ and each $N_{0 i} \times N_{1 i} \in\left\langle X_{i} \times X_{i}\right\rangle$, there exist compact $\Gamma_{i}$-convex subsets $L_{N_{0 i}}, L_{N_{1 i}}$ of $\left(X_{i} ; \Gamma_{i}\right)$ containing $N_{0 i}, N_{1 i}$, respectively, such that, for $L:=\prod_{i \in I} L_{N_{0 i}} \times \prod_{i \in I} L_{N_{1 i}}$, we have

$$
\begin{aligned}
L \backslash K \subseteq \bigcup_{\left(u_{i}, v_{i}\right) \in L_{N_{0 i}} \times L_{N_{1 i}}}\left\{\left[F_{i}^{-1}\left(v_{i}\right) \times X\right]\right. \\
\\
\left.\bigcap\left[H_{i}^{-1}\left(u_{i}\right) \bigcup\left(X \times X \backslash W_{i}\right)\right]\right\} .
\end{aligned}
$$

So, all the conditions of Theorem 30 are satisfied. Hence, by Theorem 30, there exists $(\bar{x}, \bar{y}) \in X \times X$ such that

$$
\bar{y}_{i} \in F_{i}(\bar{x}), \quad H_{i}(\bar{x}, \bar{y})=\emptyset, \quad \forall i \in I .
$$


If $\bar{x} \in V_{j}$ for some $j \in I$, then, by the definition of $H_{i}$, we have

$$
H_{i}(\bar{x}, \bar{y})=A_{i}(\bar{x})=\emptyset,
$$

which contradicts the first part of (ii). Thus, we have

$$
(\bar{x}, \bar{y}) \in X \times X \quad \text { with } \bar{x} \notin V_{i}, \forall i \in I,
$$

which implies that, for each $i \in I, \bar{x}_{i} \in B_{i}(\bar{x}), \bar{y}_{i} \in F_{i}(\bar{x})$, and $P_{i}(\bar{x}, \bar{y}) \cap A_{i}(\bar{x})=\emptyset$; that is, $(\bar{x}, \bar{y})$ is an equilibrium point of generalized abstract economy $\varepsilon$.

In Theorems 26 and 31, when $P_{i}(x, y)=P_{i}(x)$ and $F_{i}(x)=$ $X_{i}$ for each $i \in I$ and for all $(x, y) \in X \times X$, we can derive the following equilibrium existence results for abstract economies.

Corollary 32. Let I be a finite index set; let $\left\{\left(X_{i} ; \Gamma_{i}\right)\right\}_{i \in I}$ be a family of abstract convex spaces such that $(X ; \Gamma):=\left(\prod_{i \in I} X_{i} ; \Gamma\right)$ is an abstract convex space as defined in Lemma 7 . Let $\varepsilon=$ $\left(\left(X_{i} ; \Gamma_{i}\right), A_{i}, B_{i}, P_{i}\right)_{i \in I}$ be an abstract economy, and let $K$ be a nonempty compact subset of $X$. For each $i \in I$, assume that,

(i) for each $x \in X, A_{i}(x) \neq \emptyset$ and $\operatorname{co}_{\Gamma_{i}} A_{i}(x) \subseteq B_{i}(x)$;

(ii) for each $x \in W_{i}=\left\{x \in X: A_{i}(x) \cap P_{i}(x) \neq \emptyset\right\}, x_{i} \notin$ $\mathrm{Co}_{\Gamma_{i}} P_{i}(x)$;

(iii) for each $u_{i} \in X_{i}, A_{i}^{-1}\left(u_{i}\right) \bigcap\left(P_{i}^{-1}\left(u_{i}\right) \bigcup\left(X \backslash W_{i}\right)\right)$ is open in $X$;

(iv) for each $N_{i} \in\left\langle X_{i}\right\rangle$, there exists a compact $\Gamma_{i}$-convex subset $L_{N_{i}}$ of $\left(X_{i} ; \Gamma_{i}\right)$ containing $N_{i}$, such that, for $L:=$ $\prod_{i \in I} L_{N_{i}}$, we have

$$
L \backslash K \subseteq \bigcup_{u_{i} \in L_{N_{i}}}\left[A_{i}^{-1}\left(u_{i}\right) \bigcap\left(P_{i}^{-1}\left(u_{i}\right) \bigcup\left(X \backslash W_{i}\right)\right)\right] .
$$

If $(X ; \Gamma)$ satisfies $1_{X} \in \mathfrak{\Re} \mathfrak{C}(X, X)$, then there exists $\bar{x} \in X$ such that, for each $i \in I, \bar{x}_{i} \in B_{i}(\bar{x})$ and $P_{i}(\bar{x}) \cap A_{i}(\bar{x})=\emptyset$.

Corollary 33. Let I be a finite index set; let $\left\{\left(X_{i} ; \Gamma_{i}\right)\right\}_{i \in I}$ be a family of abstract convex spaces such that $(X ; \Gamma):=\left(\prod_{i \in I} X_{i} ; \Gamma\right)$ is an abstract convex space as defined in Lemma 7 . Let $\varepsilon=$ $\left(\left(X_{i} ; \Gamma_{i}\right), A_{i}, B_{i}, P_{i}\right)_{i \in I}$ be an abstract economy, and let $K$ be a nonempty compact subset of $X$. For each $i \in I$, assume that,

(i) the set $V_{i}=\left\{x \in X: x_{i} \notin B_{i}(x)\right\}$ is open in $X$;

(ii) if $x \in V_{i}$, we have $A_{i}(x) \neq \emptyset$ and $x_{i} \notin \operatorname{co}_{\Gamma_{i}} A_{i}(x)$; if $x \notin V_{i}$ with $P_{i}(x) \cap A_{i}(x) \neq \emptyset$, then we have $x_{i} \notin$ $\mathrm{Co}_{\Gamma_{i}}\left(P_{i}(x) \bigcap A_{i}(x)\right)$;

(iii) for each $u_{i} \in X_{i}$, the sets $A_{i}^{-1}\left(u_{i}\right)$ and $P_{i}^{-1}\left(u_{i}\right)$ are open in $X$;

(iv) the set $W_{i}=\left\{x \in V_{i}: A_{i}(x) \neq \emptyset\right\} \bigcup\left\{x \notin V_{i}\right.$ : $\left.P_{i}(x) \cap A_{i}(x) \neq \emptyset\right\}$ is closed in $X$

(v) for each $N_{i} \in\left\langle X_{i}\right\rangle$, there exists a compact $\Gamma_{i}$-convex subset $L_{N_{i}}$ of $\left(X_{i} ; \Gamma_{i}\right)$ containing $N_{i}$ such that, for each $x \in L \backslash K$, there exists $u_{i} \in N_{i}$ satisfying

$$
x \in\left(\left(P_{i}^{-1}\left(u_{i}\right) \bigcup V_{i}\right) \bigcap A_{i}^{-1}\left(u_{i}\right)\right) \bigcup\left(X \backslash W_{i}\right),
$$

where $L:=\prod_{i \in I} L_{N_{i}}$.

If $(X ; \Gamma)$ satisfies $1_{X} \in \mathfrak{\Re} \mathfrak{C}(X, X)$, then there exists $\bar{x} \in X$ such that, for each $i \in I, \bar{x}_{i} \in B_{i}(\bar{x})$ and $P_{i}(\bar{x}) \cap A_{i}(\bar{x})=\emptyset$.

\section{Acknowledgments}

The authors thank the referee for his many valuable suggestions and helpful comments which improve the exposition of this paper. This work was partially supported by the Planning Foundation for Humanities and Social Sciences of Ministry of Education of China (Research on utilizing conflict of water resource and initial water right allocation in a river basin-on the basis of game theory, no. 12YJAZH084) and Jiangsu Overseas Research \& Training Program for University Prominent Young \& Middle-aged Teachers and Presidents.

\section{References}

[1] E. Tarafdar, "A fixed point theorem and equilibrium point of an abstract economy," Journal of Mathematical Economics, vol. 20, no. 2, pp. 211-218, 1991.

[2] E. Tarafdar, "Fixed point theorems in $H$-spaces and equilibrium points of abstract economies," Australian Mathematical Society A, vol. 53, no. 2, pp. 252-260, 1992.

[3] Q. H. Ansari and J. C. Yao, "A fixed point theorem and its applications to a system of variational inequalities," Bulletin of the Australian Mathematical Society, vol. 59, no. 3, pp. 433-442, 1999.

[4] S. P. Singh, E. Tarafdar, and B. Watson, "A generalized fixed point theorem and equilibrium point of an abstract economy," Journal of Computational and Applied Mathematics, vol. 113, no. 1-2, pp. 65-71, 2000.

[5] W. Briec and C. Horvath, "Nash points, Ky Fan inequality and equilibria of abstract economies in Max-Plus and B-convexity," Journal of Mathematical Analysis and Applications, vol. 341, no. 1, pp. 188-199, 2008.

[6] S. Park, "Continuous selection theorems in generalized convex spaces," Numerical Functional Analysis and Optimization, vol. 20, no. 5-6, pp. 567-583, 1999.

[7] S. Park, "Generalizations of the Nash equilibrium theorem in the KKM theory," Fixed Point Theory and Applications, vol. 2010, Article ID 234706, 23 pages, 2010.

[8] S. Park, "On the von Neumann-Sion minimax theorem in KKM spaces," Applied Mathematics Letters, vol. 23, no. 10, pp. 12691273, 2010.

[9] S. Park, "The KKM principle in abstract convex spaces: equivalent formulations and applications," Nonlinear Analysis: Theory, Methods \& Applications, vol. 73, no. 4, pp. 1028-1042, 2010.

[10] S. Park, "Comments on generalized R-KKM type theorems," Communications of the Korean Mathematical Society, vol. 25, no. 2, pp. 303-311, 2010.

[11] S. Park, "Remarks on some basic concepts in the KKM theory," Nonlinear Analysis: Theory, Methods \& Applications, vol. 74, no. 7, pp. 2439-2447, 2011.

[12] S. Park, "Elements of the KKM theory on abstract convex spaces," Journal of the Korean Mathematical Society, vol. 45, no. 1, pp. 1-27, 2008.

[13] S. Park, "A genesis of general KKM theorems for abstract convex spaces," Journal of Nonlinear Analysis and Optimization, vol. 2, no. 1, pp. 141-154, 2011.

[14] C. D. Horvath, "Some results on multivalued mappings and inequalities without convexity," in Nonlinear and Convex Analysis, B. L. Lin and S. Simons, Eds., vol. 107 of Lecture Notes in 
Pure and Applied Mathematics, pp. 99-106, Dekker, New York, NY, USA, 1987.

[15] S. Park, "Fixed point theorems in locally G-convex spaces," Nonlinear Analysis: Theory, Methods \& Applications, vol. 48, no. 6, pp. 869-879, 2002.

[16] C. D. Horvath and J. V. L. Ciscar, "Maximal elements and fixed points for binary relations on topological ordered spaces," Journal of Mathematical Economics, vol. 25, no. 3, pp. 291-306, 1996.

[17] S. Park, "Abstract convex spaces, $K K M$ spaces and $\varphi_{A}$-spaces," Nonlinear Analysis Forum, vol. 17, pp. 1-10, 2012.

[18] S. Park, "Evolution of the minimax inequality of Ky Fan," Journal of Operators, vol. 2013, Article ID 124962, 10 pages, 2013.

[19] L. J. Lin, "Applications of a fixed point theorem in G-convex space," Nonlinear Analysis: Theory, Methods \& Applications, vol. 46, no. 5, pp. 601-608, 2001.

[20] J. V. Llinares, "Existence of equilibrium in generalized games with abstract convexity structure," Journal of Optimization Theory and Applications, vol. 105, no. 1, pp. 149-160, 2000.

[21] P. Prokopovych, "On equilibrium existence in payoff secure games," Economic Theory, vol. 48, no. 1, pp. 5-16, 2011.

[22] K. Fan, "A generalization of Tychonoff's fixed point theorem," Mathematische Annalen, vol. 142, pp. 305-310, 1960/1961.

[23] F. E. Browder, "The fixed point theory of multi-valued mappings in topological vector spaces," Mathematische Annalen, vol. 177, no. 4, pp. 283-301, 1968.

[24] J. Yu and G. X. Z. Yuan, "The study of Pareto equilibria for multiobjective games by fixed point and Ky Fan minimax inequality methods," Computers \& Mathematics with Applications, vol. 35, no. 9, pp. 17-24, 1998.

[25] W. K. Kim and G. X. Z. Yuan, "Existence of equilibria for generalized games and generalized social system with coordination," Nonlinear Functional Analysis and Applications, vol. 3, no. 2, pp. 77-102, 1998.

[26] M. Balaj and S. Muresan, "Generalizations of the Fan-Browder fixed point theorem and minimax inequalities," Archivum Mathematicum, vol. 41, no. 4, pp. 399-407, 2005.

[27] X. Q. Luo, "On some generalized Ky Fan minimax inequalities," Fixed Point Theory and Applications, vol. 2009, Article ID 194671, 9 pages, 2009.

[28] S. Park, "Some coincidence theorems on acyclic multifunctions and applications to KKM theory," in Fixed Point Theory and Applications, K. K. Tan, Ed., pp. 248-277, World Scientific, River Edge, NJ, USA, 1992.

[29] S. Park, "Applications of some basic theorems in the KKM theory," Fixed Point Theory and Applications, vol. 2011, article 98, 14 pages, 2011.

[30] X. P. Ding and K. K. Tan, "A minimax inequality with applications to existence of equilibrium point and fixed point theorems," Colloquium Mathematicum, vol. 63, no. 2, pp. 233247, 1992.

[31] X. P. Ding and G. X. Z. Yuan, "The study of existence of equilibria for generalized games without lower semicontinuity in locally topological vector spaces," Journal of Mathematical Analysis and Applications, vol. 227, no. 2, pp. 420-438, 1998.

[32] S. S. Chang, B. S. Lee, X. Wu, Y. J. Cho, and G. M. Lee, "On the generalized quasi-variational inequality problems," Journal of Mathematical Analysis and Applications, vol. 203, no. 3, pp. 686-711, 1996.

[33] S. S. Chang, G. X. Z. Yuan, G. M. Lee, and X. L. Zhang, "Saddle points and minimax theorems for vector-valued multifunctions on H-spaces," Applied Mathematics Letters, vol. 11, no. 3, pp. 101107, 1998.

[34] X. Wu, "Existence theorems for maximal elements in $H$-spaces with applications on the minimax inequalities and equilibrium of games," Journal of Applied Analysis, vol. 6, no. 2, pp. 283-293, 2000.

[35] Y. S. Huang, "Fixed point theorems with an application in generalized games," Journal of Mathematical Analysis and Applications, vol. 186, no. 3, pp. 634-642, 1994.

[36] P. Cubiotti and G. Nordo, "On generalized games in $H$-spaces," Commentationes Mathematicae Universitatis Carolinae, vol. 40, no. 1, pp. 175-180, 1999.

[37] Q. Luo, "Ky Fan's section theorem and its applications in topological ordered spaces," Applied Mathematics Letters, vol. 17, no. 10, pp. 1113-1119, 2004.

[38] Q. Luo, "The applications of the Fan-Browder fixed point theorem in topological ordered spaces," Applied Mathematics Letters, vol. 19, no. 11, pp. 1265-1271, 2006.

[39] N. T. Vinh, "Matching theorems, fixed point theorems and minimax inequalities in topological ordered spaces," Acta Mathematica Vietnamica, vol. 30, no. 3, pp. 211-224, 2005.

[40] L. J. Lin and Z. T. Yu, "Fixed-point theorems and equilibrium problems," Nonlinear Analysis: Theory, Methods \& Applications, vol. 43, no. 8, pp. 987-999, 2001.

[41] X. P. Ding and J. Y. Park, "Fixed points and generalized vector equilibrium problems in generalized convex spaces," Indian Journal of Pure and Applied Mathematics, vol. 34, no. 6, pp. 973990, 2003.

[42] M. Balaj and L. J. Lin, "Fixed points, coincidence points and maximal elements with applications to generalized equilibrium problems and minimax theory," Nonlinear Analysis: Theory, Methods \& Applications, vol. 70, no. 1, pp. 393-403, 2009.

[43] M. G. Yang, J. P. Xu, N. J. Huang, and S. J. Yu, "Minimax theorems for vector-valued mappings in abstract convex spaces," Taiwanese Journal of Mathematics, vol. 14, no. 2, pp. 719-732, 2010.

[44] W. K. Kim and K. K. Tan, "New existence theorems of equilibria and applications," Nonlinear Analysis: Theory, Methods \& Applications, vol. 47, no. 1, pp. 531-542, 2001.

[45] L. J. Lin, Z. T. Yu, Q. H. Ansari, and L. P. Lai, "Fixed point and maximal element theorems with applications to abstract economies and minimax inequalities," Journal of Mathematical Analysis and Applications, vol. 284, no. 2, pp. 656-671, 2003.

[46] L. Wang, N. J. Huang, and C. S. Lee, "Some new existence theorems of generalized abstract fuzzy economies with applications," Taiwanese Journal of Mathematics, vol. 14, no. 1, pp. 47-57, 2010.

[47] T. C. Lai, Y. C. Lin, and J. C. Yao, "Existence of equilibrium for abstract economics on pseudo $H$-spaces," Applied Mathematics Letters, vol. 17, no. 6, pp. 691-696, 2004. 


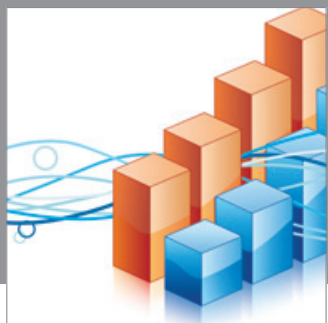

Advances in

Operations Research

mansans

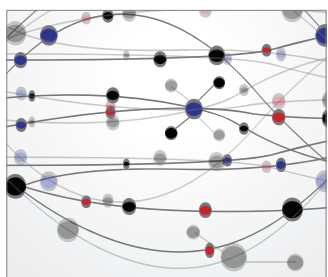

The Scientific World Journal
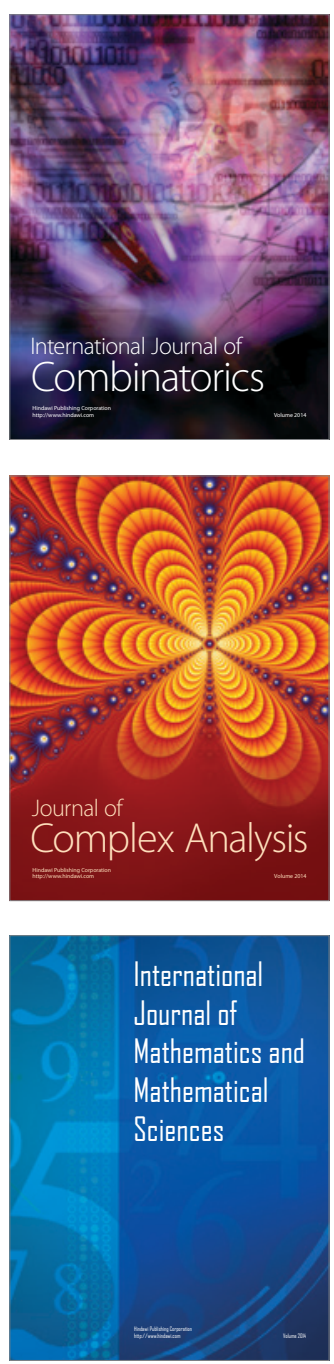
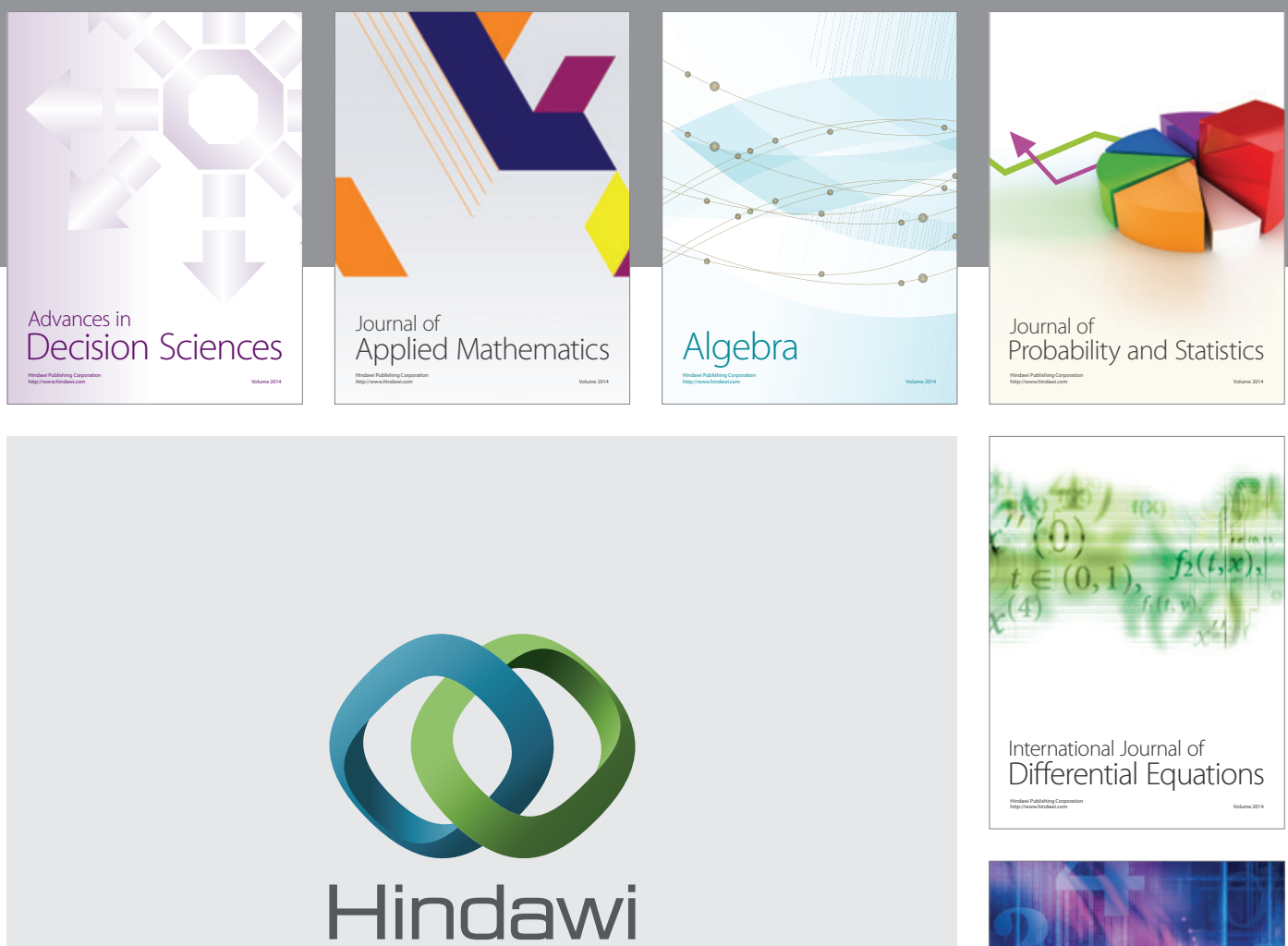

Submit your manuscripts at http://www.hindawi.com
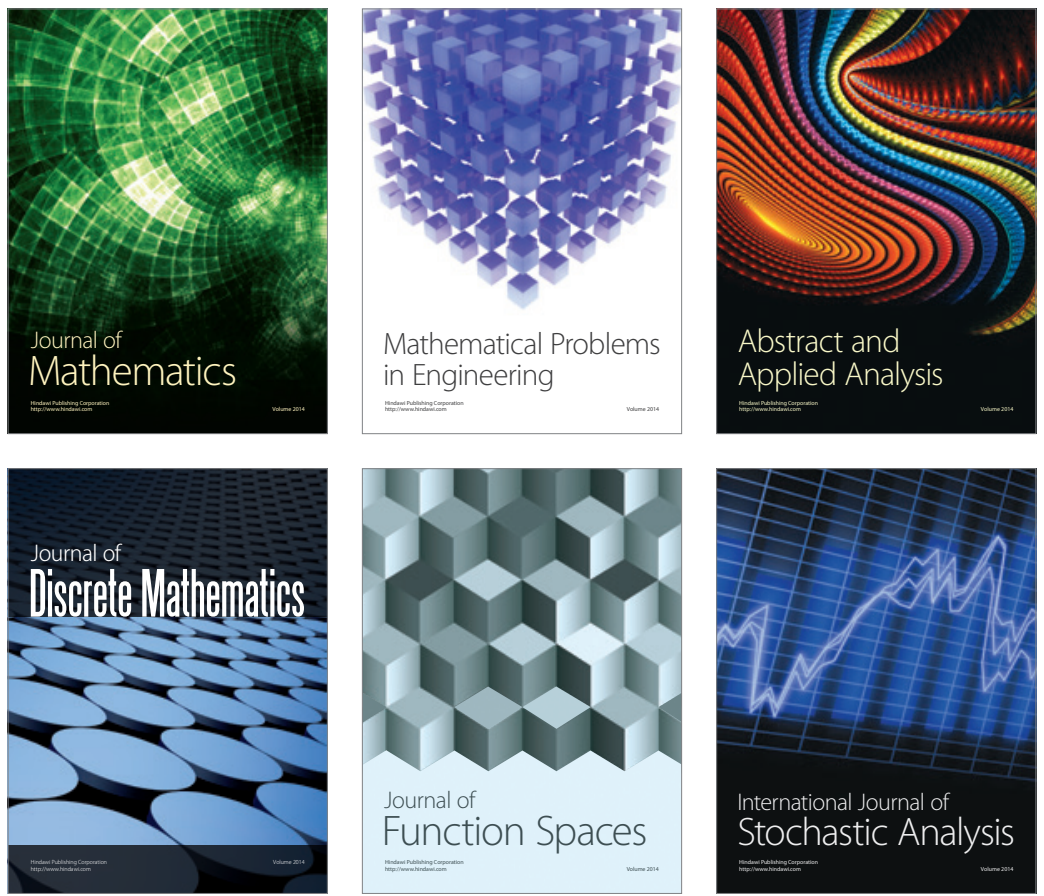

Journal of

Function Spaces

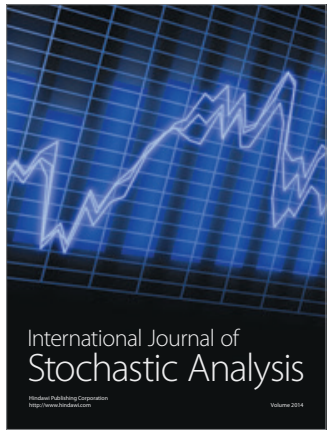

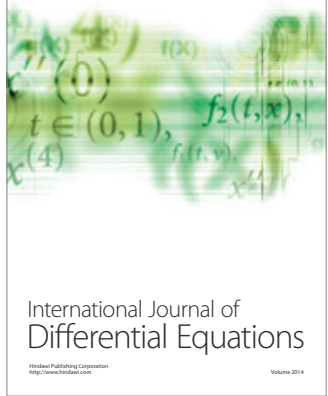
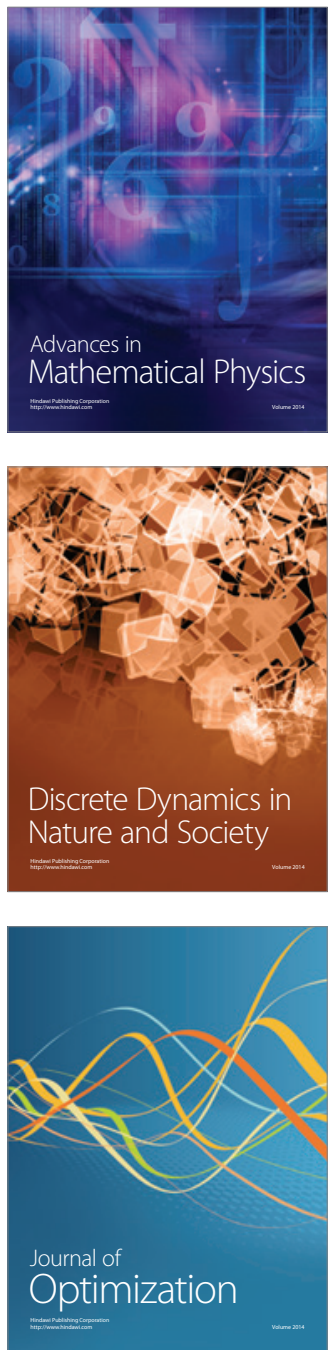